\title{
La folie du dire dans « la trilogie » de Ben Jelloun
}

Résumé: La littérature maghrébine francophone est essentiellement hybride, entre langues, grammaires, et traditions artistiques et littéraires. Dans le but d'exprimer cette hybridité, les écrivains sont prodigues dans l'utilisation de stratégies intertextuelles : ils font cohabiter, sur la même page, versets du Coran, proverbes berbères, citations de poètes et de philosophes de l'Orient comme de l'Occident, et éléments du quotidien de l'immigré ou de l'exilé dans les grandes métropoles européennes, dans un amalgame de cultures, de sociétés et de civilisations. Les textes de Tahar Ben Jelloun reproduisent cette mosaïque culturelle et composent un complexe kaléidoscope de références, fidèles ou non, qui oscillent entre divers registres implicites d'ordre culturel, artistique ou spécifiquement littéraire. Dans les trois romans considérés ici comme une trilogie, L'Enfant de sable (1985), La Nuit sacrée (1987, Prix Goncourt) et La Nuit de l'erreur (1997), Ben Jelloun met en mouvement le kaléidoscope de l'hybridité culturelle en ayant recours à un narrateur exceptionnel : le(s) conteur(s) d'histoires.

Mots clés : Tahar Ben Jelloun, conteur d'histoires, récit, narration, métarécit

À son arrivée à Paris, en 1971, le Marocain Tahar Ben Jelloun (1944) portait dans sa valise deux titres de poésie publiés à Casablanca, un diplôme de journalisme, et un projet de thèse de doctorat en psychiatrie sociale sur la sexualité de l'immigré maghrébin en France. Devenu collaborateur du journal Le Monde, il fait publier de nouveaux poèmes, ainsi que sa thèse (sous forme d'essai, de roman et d'articles de presse). Toujours présent dans les médias, en peu de temps il devient un important porte-parole des immigrés maghrébins en France et s'engage dans la tâche de parler pour et à l'immigré en terre d'exil. En quelques années, il est rapidement considéré comme l'un des plus éminents intellectuels maghrébins en Europe.

Du moment où elle donne une voix et/ou la parole à l'immigré en terre d'accueil et d'exil, son œuvre est imprégnée de deux caractéristiques singulières : 1) elle enregistre par écrit traditions, contes et récits oraux - en réalisant un travail essentiellement hybride, sur divers niveaux ; et 2) elle veut faire le portrait de l'immigré comme un être non-exotique, renforçant pourtant sa culture « autre », diverse ; cette littérature se revêt donc d'étrangeté - qualité d'être à la fois étrange et étranger, oscillant entre géographies, registres et genres. 
Nous allons parcourir, sur les pas du personnage emblématique du conteur d'histoires - alter ego de l'auteur et narrateur privilégié dans les trois romans qui constituent ici notre corpus -, le trajet qui relie ces caractéristiques singulières au caractère métanarratif de la prose benjellounienne, fondée sur le semblant d'oralité et l'impression tragique de la perte.

\section{L'hybridité}

D’une façon générale, la littérature maghrébine francophone s'exprime dans le pays d'adoption à propos de thèmes et questions qui ont trait à la terre d'origine. Territoire donc ambivalent, d'entre-deux, cette littérature est inévitablement hybride entre langues, grammaires, et traditions artistiques et littéraires.

Dans le but d'exprimer cette hybridité, les écrivains sont prodigues dans l'utilisation de stratégies intertextuelles : ils font cohabiter, sur la même page, versets $\mathrm{du}$ Coran, proverbes berbères, citations de poètes et de philosophes (de l'Orient comme de l'Occident), et éléments du quotidien de l'immigré ou de l'exilé dans les grandes métropoles européennes, dans un amalgame de cultures, de sociétés et de civilisations. Dans leur ensemble, les textes de Tahar Ben Jelloun reproduisent cette mosaïque culturelle et composent un complexe kaléidoscope de références implicites, fidèles ou non, oscillant entre divers registres, et d'ordre culturel, artistique ou spécifiquement littéraire.

Dans trois de ses romans, L'Enfant de sable (1985), La Nuit sacrée (1987 ; Prix Goncourt) et La Nuit de l'erreur (1997), Ben Jelloun met en marche le mécanisme intertextuel par l'intermédiaire d'un narrateur exceptionnel : le conteur d'histoires. En fait, il s'agit plutôt d'une succession de conteurs, qui se disputent le tour et la parole en pleine place publique dans l'exercice de la narration - réalisant un hybridisme complémentaire entre narration et récit, et entre contes oraux et écrits. Dans l'interaction des différents conteurs, et entre conteurs et auditeurs dans la dispute pour la version de l'histoire d'Ahmed/Zahra (dans les deux premiers romans) ou de Zina (dans le troisième), les liens de l'histoire se tissent et s'embrouillent. C'est la présence centrale du conteur dans ces trois romans qui les relie et leur accorde une unité de premier abord thématique, mais également structurale.

Le succès phénoménal du premier roman a généré sa continuation dans le deuxième. Le troisième a été écrit entre 1991 et 1996 (information qui clôt le récit). Dans une entrevue (Ben Jelloun 1998), l'auteur explique qu'il a " souffert » pour réussir à terminer ce livre, tâche qu'il s'était imposée depuis la publication de $L a$ Nuit sacrée (ce qui augmente encore plus l'écart chronologique entre ces romans, tout en rapprochant pourtant leurs projets d'écriture). Le laps de dix ans entre la 
parution de La Nuit sacrée et de La Nuit de l'erreur révèle que l'on a affaire à une drôle de trilogie, puisque entre les deux Nuits (dont la similitude des titres renforce l'association), Ben Jelloun a publié 11 autres titres ( 9 de prose et 2 de poésie).

Le personnage du conteur d'histoires avait déjà fait son apparition dans d'autres récits de Ben Jelloun, mais de façon accessoire et figurative. Ici, même quand il y a une succession de conteurs, son poste demeure comme un élément axial de l'engrenage du récit. L'Enfant de sable, texte polyphonique où l'exubérance des conteurs et de leur succession obscurcit l'éventuelle conclusion de l'histoire du protagoniste, est suivi par le récit relativement linéaire de La Nuit sacrée, roman qui confère enfin un destin à l'hérö̈ne (devenue conteuse à son tour). La Nuit de l'erreur aura d'autres personnages, mais la succession des conteurs revient sur la place publique, lieu où se passe la narration de l'histoire ou des histoires, restaurant la polyphonie de L'Enfant de sable. C'est comme si L'Enfant de sable avait deux suites possibles : l'une qui reste cohérente par rapport à l'histoire entamée, mais laissée en suspens ( $L a$ Nuit sacrée), et l'autre qui reprend la structure narrative polyphonique, polysémique et fragmentaire (La Nuit de l'erreur). L'Enfant de sable, texte foncièrement hybride, est donc un récit dont les sentiers bifurquent.

\section{Personnages de l'entre-deux}

Alter ego idéal de cet écrivain qui s’investit de la fonction de porte-parole, le personnage-narrateur du conteur d'histoires est un élément particulièrement stratégique dans l'élaboration de la tessiture de différents registres dans le texte. Ceci constitue un élément important en ce qui concerne la mémoire de la collectivité maghrébine, puisque le conteur réalise, jusqu'à nos jours, son métier millénaire auprès des populations illettrées. Même s'il fait partie de la tradition littéraire et culturelle de l'Orient, cette tradition est comparable à celle de l'Europe médiévale, étudiée par Paul Zumthor (1987), qui définit la situation du conteur : il s'agit d'un être réalisant des échanges intervocaux mobiles, qui reçoit des contributions lors des interventions dialogiques de son public, tout en les faisant circuler par le biais de son itinéraire nomade (Zumthor 1987, 70ss) ; à partir de ces apports, le conteur crée des variations aux histoires déjà racontées, déjà connues sans pourtant être reconnues, du fait de la pratique de l'improvisation, inhérente à la tradition orale. Le déplacement du conteur est le garant du déplacement de la mémoire, de l'histoire et du texte. Le conteur maghrébin est, dans ce sens, comparable au jongleur médiéval, dont le nomadisme exceptionnel perpétue la circulation et la transmission des histoires et des traditions.

Cependant, outre la diversité apparente, le phénomène de l'unité s'impose : 
Dans le kaléidoscope du discours que tient, sur la place du marché, à la cour seigneuriale, sur le parvis de l'église, l'interprète de poésie, ce qui se révèle à ceux qui l'entendent c'est l'unité du monde. Les auditeurs ont besoin d'une telle perception [...] pour survivre. Elle seule, par la grâce d'une parole étrange, fait sens, c'est-à-dire rend interprétable ce que l'on vit. (Zumthor 1987, 81-82)

Ainsi, l'histoire d'Ahmed serait, selon le commentaire de l'un des auditeurs (participatifs) de L'Enfant de sable, une variation de la légende du guerrier Antar (Ben Jelloun 1985, 83), une autre femme travestie dont le récit surgit en abyme dans celui sur Ahmed. Il s'agit du thème littéraire de la jeune fille élevée comme un garçon, qui existe dans plusieurs traditions littéraires et dont la répétition assure la compréhension et la reconnaissance de la légende. Cette compréhension est surtout, selon Zumthor, l'assurance de la reconnaissance de soi à l'intérieur de la collectivité ; il est également l'élément mobilisant « des capacités inventives de populations que rien autrement n'eût rapprochées » (Zumthor 1987, 78), les économiquement défavorisés et les illettrés, qui, du fait de leur condition, ne pourraient s'intégrer au mouvement de la dynamique culturelle.

Selon cette perspective, les histoires d'Ahmed/Zahra et de Zina, étendues de l'échelle personnelle à celle de la collectivité, peuvent être comprises comme emblématiques de toute une société agressée, violée, travestie ; elles permettent aux auditeurs de repenser leur propre identité individuelle, ainsi que celle de leur société.

La pluralité des conteurs opère pourtant, surtout, dans le sens d'un ébranlement de l'idée de "vérité » ou de véracité de la version ou de l'histoire, ainsi que de l'autorité monologique, dans une écriture métatextuelle caractéristique de la prose de Ben Jelloun. Comme il sied à un texte contemporain, l'importance de l'interprétation lectorale ouvre la voie aux diverses médiations des innombrables conteurs et auditeurs participatifs, en donnant au lecteur voix au chapitre. Ainsi, l'entre-deux d'Ahmed-Zahra, homme-femme, crée justement un personnage qui n'est ni l'un ni l'autre, ou les deux en même temps, comme dans l'androgynie mythique, qui surmonte les divisions et devient quelque chose de plus vaste, emblème du collectif, prétexte à ce que chaque conteur l'approche de ses propres idées de violence, d'exil ou de perte. Le protagoniste peut ainsi être vu comme métaphore du livre : dans les frottements des paroles proférées, Ahmed, corps travesti, et Zina, corps violé, demeurent ouverts à la multiplicité du regard lectoral. 


\section{L'étrangeté}

Ce protagoniste, emblème de la société et métaphore du livre (selon une perspective métatextuelle) et ce narrateur-conteur à la croisée de différentes traditions littéraires sont tous les deux des personnages essentiellement tragiques. Ce tragique n'est pas seulement un élément constitutif du héros exemplaire (selon une perspective classique) ; il est de plus une marque, une empreinte de la littérature issue de l'immigration. Dans la mesure où cette littérature met en scène la thématique de l'émigration, elle renvoie inexorablement aux questions de l'exil, de l'éloignement, de l'isolement et de la rupture - différentes déclinaisons du thème tragique de la perte.

Le critique Charles Bonn met ainsi en relief cet aspect tragique de la littérature issue de l'immigration :

Métaphore tragique de l'écriture[,] [...] l'émigration n'est donc pas un thème comme les autres. [...] Elle signale le tragique d'une écriture qui ne peut se développer que dans et par son étrangeté, et ne peut dire l'espace clos de l'oralité ${ }^{1}$ dans l'ouverture de la lecture romanesque, qu'en y introduisant la rupture, la perte. (Bonn 1994, 53)

Autre perte : ce paradoxe tragique qui conditionne la transmission de toute une culture (maghrébine) de l'oralité, selon les codes (européens) du roman, du texte écrit. Le tragique et l'étrangeté sont, de ce point de vue, des aspects contigus de la littérature issue de l'immigration, lesquels constituent la contradiction intrinsèque de devoir s'exprimer «sans étrangeté », en essayant de présenter l'immigré au lecteur européen ou occidental comme un interlocuteur non-exotique, mais, en même temps, " par l'étrangeté », justement parce qu'il est autre, divers, porteur d'une autre parole, d'un autre discours, d'un autre regard et en dehors de la tradition orale.

Le thème de l'émigration se manifeste, dans notre corpus, sous la forme des errances et déplacements constants des conteurs d'histoires. À ce propos également, Bonn nous donne de précieux indices :

L'errance du sens qui depuis l'antiquité apparente le barde au devin comme au fou, et s'y manifeste par exemple dans l'exterritorialité de Tirésias chez Sophocle, ou dans la cécité d'Homère selon le mythe, se double ici du saut entre deux mondes, où le lieu d'où l'on se réclame ne vaut que par rapport à l'ailleurs, à l'autre. (Bonn 1994, 54-55)

1 L'économie de cet article ne permet pas de développer ici le commentaire sur l'intérieur clos et protégé des demeures, lieu privilégié de la conteuse d'histoires, par opposition à l'espace ouvert de la place publique où prédomine le conteur. 
Les errances du barde et du sens ont trait à la contingence de la littérature maghrébine d'expression française, qui est d'exister en tant que résultat d'un changement de territoire, et de se définir en fonction de ce déplacement, dans le rapport d'altérité. «L'ailleurs » et « l'autre » sont ce dont on parle, et la distance est le lieu même d'où on parle. Cette littérature se crée ainsi dans le laps et dans l'absence. Elle exprime et réitère la condition d'entre-deux de l'immigré ; elle manifeste, par l'écriture, le caractère double et contradictoire de l'adoption d'une tradition " étrangère » - le romanesque ; elle surimpose, dans les pas des personnages errants, l'impression et l'empreinte de la perte, mais aussi de la quête d'une nouvelle identité et de nouveaux sens à frayer.

\section{La folie des conteurs de Ben Jelloun}

La fascination de Ben Jelloun pour l'image millénaire du conteur d'histoires le mène à faire du conteur qui occupe la place centrale de Marrakech, Djema el Fna, un narrateur privilégié dans les trois romans cités. Et, dans chacun des romans, ces êtres errants sont, pour reprendre les termes de Bonn, des « bardes apparentés aux devins et aux fous ». Dans L'Enfant de Sable, par exemple, Bouchaỉb devient fou à cause du secret et du livre qui le contenait ; dans La Nuit Sacrée, Zahra vit le cauchemar de la Sainte des sables parce qu'elle n'a pas su se dégager des histoires qu'elle avait accumulées ; dans La Nuit de l'Erreur, Dahmane, aliéné, quitte la place afin de pouvoir lire le manuscrit de Zina et le réécrire ; et Zina, dans l'une de ses projections, se travestit en Moha (personnage entre fou et bouffon, qui ressurgit occasionnellement dans les romans de Ben Jelloun) et dénonce des injustices en pleine place publique. Tous ces conteurs hallucinés, délirants, aliénés ou fous ont une conscience exceptionnelle et diverse des choses. Et, dans l'inversion qui les caractérise, ils ont la possibilité de parler aux puissants, et de réaliser une critique de la société sous l'apparence d'une rhétorique étourdie.

Les personnages de ces conteurs forment essentiellement deux groupes. Le premier, les protagonistes, sont des êtres tragiques et solennels, et poursuivent une trajectoire classique de quête, apprentissage et rédemption. Le second, les personnages secondaires, constituent un ensemble hétéroclite d'être grotesques : ceux qui entourent Bouchaïb le hantent comme une parade, fantasmatiques, exigeant qu'il apporte une fin à l'histoire. Ceux de Zahra, également en pèlerinage, lui demandent une guérison dans un enfer lumineux dans le désert ; ou alors, des mendiants morts et suspendus en l'air, qui la supplient de révéler leur image emprisonnée et oubliée ; ou encore des enfants déchaînés contre le monde et le temps, réfugiés dans le mirage d'une oasis. Ceux de l'histoire de Zina sont des 
êtres de rêve - borgnes aux tics nerveux, musiciens manchots, nains marchant sur les mains, aveugles feignant de lire, femmes obèses et patriarches muets (1997, 16) ; ou alors, les hommes du café, ridicules dans leurs velléités mondaines ; outre les innombrables hordes ambulantes de déshérités, de figurants qui peuplent la scène en silence dans une mise en scène baroque, étourdissante et confuse, comme ceux qui attendent dans le port de Tanger le jour fictif d'un embarquement défendu.

Ben Jelloun révèle une vraie prédilection pour ces personnages dépossédés et voués au désespoir. Ils constituent la matière première des conteurs, eux aussi (chacun à sa manière) marginaux. Dans cette superposition de dépossessions, l'écrivain exprime, de façon cinglante, la douleur de l'exil et du déterrement. Les conteurs et leurs personnages, pour la plupart excessifs, felliniens et circassiens, expriment cette douleur par leur caractère exceptionnel, pathétique et tragique.

Le conteur exprime également la douleur inhérente au conte même. Et quand il raconte son histoire, il se laisse pénétrer et dominer, et devient un avec le livre. Dans L'Enfant de sable, Bouchaib dit : « je sus alors que j'étais en possession d'un livre rare [... je l'ai lu]. Je suis ce livre. Je suis devenu le livre du secret[,] [...] j'ai senti le livre s'incarner en moi » $(1985,12-13)$. Le troubadour aveugle affirme : « on aurait dit que j'étais dans un livre[,] [...] j'étais peut-être un livre » (1985, 177-178). L'un des conteurs décrits par Zahra dans le début de La Nuit sacrée, annonce : « je viens d'une saison hors du temps, consignée dans un livre, je suis ce livre » $(1987,17)$.

« Devenir le livre » signifie également être un réceptacle d'histoires. Bouchaïb dit : « les histoires viennent à moi, m’habitent et me transforment. J'ai besoin de les sortir de mon corps pour libérer des cases trop chargées et recevoir de nouvelles histoires » (1985, 15-16). Ainsi, le métier du conteur exige non seulement de lui le mouvement itinérant et circulaire dans l'acquisition et la transmission d'histoires (comme on l'a vu) ; il doit être aussi un lieu de passage, le véhicule d'une transhumance interne : histoires et personnages qui envahissent le conteur et l'habitent jusqu'à ce qu'il ait besoin de les expulser de son intérieur, pour pouvoir perpétuer la circulation des histoires. Cette idée fait du conteur un exorciste singulier, qui doit à la fois libérer les personnages et se dégager de leurs histoires. Le possesseur du texte devient ainsi, étrangement, un possédé du texte.

Zahra, par exemple, dira : " tous les personnages que j'avais accumulés durant ma vie étaient priés de quitter les lieux. Je les expulsais sans hésiter » $(1997,174)$. Et Zina dira à son tour : « il faut que je raconte cette histoire. Il faut que je me vide » (1997, 13). Dahmane, dans le même sens, expliquera à l'audience : «Ô mes amis, je ne suis qu'un conteur manchot dépositaire d'une histoire qui me trouble [...] depuis que cette histoire s'est versée en moi, je me sens possédé » $(1997,111)$. 
Mais c'est Bouchaïb le plus possédé des exorcistes benjellouniens : « je tente [d'ouvrir le manuscrit] et de le délivrer des mots[,] [...] il me possède, m’obsède et me ramène à vous » $(1985,108)$. Le livre s'incarne dans le conteur afin d'être transmis, animé, revivifié, au point de l'affoler :

Je me retrouvais ainsi avec des bouts d'histoire, empêché de vivre et de circuler. Mon imagination était ruinée. [...] Je radotais. Je bégayais. Je n'étais plus un conteur, mais un charlatan, un pantin entre les doigts de la mort. [...] Les personnages que je croyais inventer [...] m'interpellaient et me demandaient des comptes. (1985, 203-204)

Possédé, effaré, immobilisé, aphasique, marionnette suspendue aux fils de sa propre histoire, Bouchaïb se perd et s'isole dans les déserts du Sud et se laisse dominer par le livre - jusqu'à ce que la pleine lune vide les pages, libère le texte et lui rende des feuilles blanches. Épuisé, le conteur abandonne son métier et part réciter le Coran dans les cimetières.

Étrangement donc le livre s'empare du conteur et lui transmet son histoire, mais pas son autorité (symbolique, conventionnellement attribuée au texte écrit) ; il lui transmet en revanche le fardeau d'une " narration définitive » (comme l'est le texte écrit). Or, de ce contresens - car les narrations sont par nature variées, distinctes, improvisées et fabulées au gré du plaisir de la dynamique de la profération - surgit l'exigence de l'exorcisme, de l'expurgation de la vérité contenue dans le livre, dont le conteur ne réussit pas à s'approprier par son processus créatif habituel.

En lui imposant cette purge, le livre usurpe son autorité de figure emblématique de la tradition de la littérature orale ; il le désemplit de son ethos en l'emplissant de logos ; il séquestre sa voix (y compris comme volonté) - qui est précisément son instrument de travail, dont la maîtrise est une condition essentielle à la perpétuation de sa tradition.

De plus, comme il sied aux textes contemporains, l'autorité du conteur est mise en question par la cacophonie de voix - les divers narrateurs, entre conteurs et auditeurs qui interviennent dans la narration ; polyphonie qui révèle la stratégie textuelle benjellounienne, où "l'unité du monde » se révèle chimère ou trompe l'œil, folie devant être exorcisée.

\section{La folie du dire}

Le conteur occupe une halqa parmi plusieurs. Son histoire est l'une de tant d'autres qui sont racontées en concomitance. Si son histoire déplaît à l'audience, celle-ci se disperse et se déplace vers une autre halqa, comme dans le kaléidos- 
cope de Zumthor. Les auditeurs grouillent autour de cercles tangents, en perpétuel mouvement de réunion et désagrégation. Et il n'y a pas que des conteurs, il y a également des acrobates, guérisseurs, saltimbanques, boxeurs, dresseurs de singe, charmeurs de serpents, écrivains publics, vendeurs d'eau, danseurs, musiciens, sorciers, aveugles, mendiants, femmes affairées, vieillards, et des enfants, beaucoup d'enfants. Cette multiplicité déborde l'espace scénique de la place publique et la transforme en carrefour de tout un patrimoine historique, social, économique et culturel.

On peut considérer la place Djema el Fna comme le lieu par excellence de la médiation, où plusieurs médiateurs (parmi lesquels les divers conteurs) essayent d'attirer l'attention d'un public mobile et instable. Lieu de la prédominance de l'oral sur l'écrit, elle est le choix de Ben Jelloun en tant que lieu de la narration dans un récit, ce qui favorise la réverbération de la tradition de la littérature orale arabe.

La place publique est le principal lieu occupé par le conteur, mais dans L'Enfant de sable, à la disparition de Bouchaïb, les membres de l'audience qui prennent la parole le font dans un café, autre espace annexe à la place publique, dont l'inclusion dans la trame marque une nouvelle étape de la narration dans le roman.

La Nuit sacrée connaît aussi ce glissement de l'activité narratrice de la place publique vers l'intérieur clos et protégé - d'un hammam, d'une demeure et d'une prison, dans des ruptures claires et successives dans l'évolution de la trame.

Dans La Nuit de l'erreur, l'histoire est racontée dans une ou plusieurs places non identifiées, puisque le couple de conteurs se déplace d'une ville à l'autre en camionnette. En arrivant à Marrakech, Jamila s'installe à Djema el Fna. Mais l'action se déroule à partir des cinq hommes qui sont dans le café - le Café de Paris qui devient, sans aucune explication, le Café Cristal. Fragmentaire et circulaire, l'action commence et termine, constamment reprise autour de la table : « Si la terre est ronde, notre histoire l'est aussi [...] Rien ne s'arrête, tout recommence : les histoires comme ceux qui les racontent » $(1997,246)$.

Le café, comme la place, accueille une multiplicité insolite :

La porte que j'ouvre aujourd'hui donne sur un café, un lieu sans mystère. Pourtant, je ne comprends pas pourquoi on a accroché sur les murs des pancartes où des phrases ont été calligraphiées en arabe : " Allah », " Mohamed ", " Nous sommes à Dieu et à Lui nous retournons » [...] « La maison ne fait pas de crédit », « Buvez Coca-Cola » [...] « La souffrance est humaine ». (1997, 245)

Affiches, paperolles, annonces, avertissements : une pléthore de textes recouvre les murs du café dans un kaléidoscope coloré et amusant, qui inclut les noms de Dieu et du prophète, des extraits du Coran, et la phrase qui a failli être choisie 
comme titre du livre ( « la souffrance est humaine », Ben Jelloun 1998, 43; phrase répétée par Zina dans un dialogue ; 1997, 270). Lieu donc, contrairement à ce qu'annonce le texte, empreint d'un certain mystère.

Le conteur, dans sa relation dialogique avec l'audience, s'intègre à l'univers cacophonique et polyphonique de la place publique, et le nourrit avec son propre caractère emblématique. Le fait d'être le narrateur principal d'une histoire le met dans une sorte de mise en abyme par rapport à la fonction narratrice. Un conteur dans la peau d'un narrateur, ou vice-versa, réalise le dédoublement de l'identité narratrice et la met en discussion, métatextuellement. Le conteur rajoute sa présence d'orateur à la narration. Son discours se destine à être entendu et, même retranscrit dans le texte narratif, toute une composante rhétorique transporte le lecteur aux rangs de la halqa. On simule ainsi une vocalité que le texte de Ben Jelloun évoque avec insistance.

Le critique Najib Redouane a mis en relief cette caractéristique, qu'il appelle « semblant d'oralité ». Selon lui, le texte de Ben Jelloun est marqué par ce phénomène :

L'écrivain exprime une tendance à cultiver le mythe de l'existence d'un modèle d'écriture fondé sur une composition poétique, une recherche esthétique, une structure rythmique et une atmosphère onirique. C'est une stratégie subtile qui cherche à séduire et à amener le lecteur à adopter à l'égard des choses racontées une attitude fondée sur la croyance et la communion. (Redouane 1994, 77)

Une telle stratégie se fonde donc sur le pouvoir évocatoire de l'oralité, ou, si l'on veut, de l'effet d'oralité de l'écriture de Ben Jelloun. Par contre, la « communion » procurée n'est rien d'autre que l'insertion de l'optique du lecteur individuel dans l'ensemble, imaginaire ou projeté, des auditeurs réunis en cercle autour du conteur, à partir du moment où ce lecteur est convoqué à écouter, de près, l'histoire racontée presque en secret, en sourdine, selon le pacte de complicité qu'inculque le conteur. On peut en donner un exemple assez frappant :

Amis du Bien, sachez que nous sommes réunis par le secret du verbe dans une rue circulaire, peut-être sur un navire et pour une traversée dont je ne connais pas l'itinéraire. [...] Que ceux qui partent avec moi lèvent la main droite pour le pacte de la fidélité. Les autres peuvent s'en aller vers d'autres histoires, chez d'autres conteurs. Moi, je ne raconte pas des histoires pour passer le temps. [...] J'ai besoin de vous. Je vous associe à mon entreprise. Je vous embarque sur le dos et le navire. (1985, 15-16 ; c'est moi qui souligne)

Cet extrait du discours poétique, prophétique et inaugural du conteur qui domine le récit et la narration de L'Enfant de sable contient une phrase prononcée par l'auteur dans une entrevue accordée deux ans avant la publication du roman : « Mes objectifs ? Témoigner. Dire. Essayer de ne pas trahir l'écoute. Je ne raconte pas 
des histoires pour passer ou faire passer le temps dans le train » (Ben Jelloun 1983, 44 ; c'est moi qui souligne). Cette « coïncidence » scelle l'identité entre l'auteur et le narrateur, et leur vocation, en tant que conteurs, de médiateurs ou passeurs culturels. Les deux extraits parlent de la narration comme une sorte de voyage qui n'a rien de divertissant ou d'anodin : l'écrivain-conteur " ne raconte pas des histoires pour passer le temps »; il tisse patiemment, progressivement, de fil en aiguille, de roman en roman, les liens entre ses plaquettes de poésie et sa thèse (qu'il porte avec lui et avec soin dans ses valises, à son arrivée à Paris en 1971, comme nous l'avons dit au début de cet article) ; il nous fait embarquer dans sa prose poétique et nous montre (ou dénonce), sous les diverses facettes des différentes figures de plusieurs conteurs, le visage symbolique et douloureux de l'émigré, de l'être qui vit ou survit entre deux mondes.

\section{En guise de conclusion}

La littérature maghrébine francophone constitue un territoire de l'autre, où l'on écrit par allusion à l'oralité et à l'exil. Ce territoire est également tragique, puisqu'il s'agit d'une littérature de l'errance et de l'immigration, marquée et imprégnée par les ruptures qui lui sont inhérentes.

L'étude de la trilogie de Ben Jelloun permet de constater que le caractère hybride et l'étrangeté de l'écriture (tels que décrits ci-dessus) s'ajoutent au semblant d'oralité du texte. Par ce moyen l'auteur se dédouble, s’incarne dans l'image millénaire du conteur d'histoires, et « dit par écrit », en transgressant les codes du genre, en traversant les versants des flux migratoires, en oscillant entre les traditions littéraires et en donnant voix à des personnages qui, d'habitude, n'en ont pas dans leurs sociétés.

À travers la pléthore de conteurs possédés et de personnages dépossédés, Ben Jelloun fait parler, dans une vraie cacophonie, tout un patrimoine culturel, une mémoire collective, en la dévoilant au lecteur qui se laisse entraîner dans ce voyage dans le temps et dans l'espace... du livre.

\section{Bibliographie}

Ben Jelloun, Tahar. «La Réclusion de l'écrivain ». L'Afrique Littéraire (Romans maghrébins, 1970-1983) nº 70 (1983) : 41-45.

Ben Jelloun, Tahar. L'Enfant de sable. Paris : Seuil, 1985.

Ben Jelloun, Tahar. La Nuit sacrée. Paris : Seuil, 1987. 
Ben Jelloun, Tahar. La Nuit de l'erreur. Paris : Seuil, 1997.

Ben Jelloun, Tahar. «Tahar Ben Jelloun : La Nuit de l'erreur ». Entretien accordé à Akalay Lotfi. La Vie Économique (Maroc). http://www.marocnet.net.net.ma/vc3901/p43.html, [1998] : 43 (26 juin 1998).

Bonn, Charles. «Le voyage innommable et le lieu de dire : émigration et errance de l'écriture maghrébine francophone ». Revue de Littérature Comparée n 1 (1994) : 47-59.

Redouane, Najib. «La stratégie esthético-stylistique de l'écriture de Ben Jelloun ». Journal of Maghrebi Studies, vol. 2 nº 1 (1994): 64-79.

Zumthor, Paul. La Lettre et la voix. De la littérature médiévale. Paris : Seuil, 1987.

Luciana Persice Nogueira is a professor of French language and French language literatures at Rio de Janeiro State University. Her Ph.D. was published under the title "Le thêâtre du conteur d'histoires de Tahar Ben Jelloun" (http:// www.limag.refer.org/Theses/Nogueira.PDF, 2001, 250p.). Her other publications include "Proust et sa vision de Ruskin : rétrospection et passage entre les 'deux côtés'”. Cahiers de l'Association Internationale d'Études Françaises nº6 (2014). Paris. 279-305 (awarded the Prix de l'AIEF pour l'Amérique latine in 2013). She is a member of three Brazilian research groups on contemporary literature. 